\title{
Research on Electromagnetic Detection Emission System for Spread Spectrum Code
}

\author{
Shiqiang $\mathrm{Li}^{1}$, Guoqiang $\mathrm{Liu}^{1,2, *}$, Yaning $\mathrm{Han}^{1,3}$, Yanhong $\mathrm{Li}^{1}$ and $\mathrm{Xing} \mathrm{Xu}^{1,3}$ \\ ${ }^{1}$ Institute of Electrical Engineering, Chinese Academy of Sciences, 100190 Beijing, China \\ ${ }^{2}$ University of the Chinese Academy of Sciences, 100049 Beijing, China \\ ${ }^{3}$ Hebei University of Technology, 300132 Tianjin, China \\ ${ }^{*}$ Corresponding author
}

\begin{abstract}
The goaf under the transmission line is prone to natural disasters such as surface subsidence and uneven surface subsidence, which poses a great threat to the surface power facilities. The effective exploration and evaluation of goaf can greatly reduce the probability of occurrence of accidents. The goaf under the transmission line has a complicated electromagnetic environment compared with the general goaf. Traditional methods, such as high-density resistivity method and transient electromagnetic method, usually increase transmitted power to improve signal-to-noise ratio, which will make the instrument bulky and not conducive to detection in wild. Based on spread spectrum code technology in communication field, an electromagnetic detection emission system is studied and designed by widening the frequency of transmitted signal and combining the correlation algorithm of spread spectrum, which can achieve better anti-interference ability without increasing power and improve the detection efficiency and accuracy.
\end{abstract}

Keywords-transmission line; goaf; spread spectrum code; electromagnetic detection emission system

\section{INTRODUCTION}

The exploitation of coal and other mineral resources has left a large number of goaf areas, which is prone to natural disasters such as surface subsidence and uneven surface subsidence, the non load stress is likely to cause the power components damage, fracture, deformation and other accidents, which poses a great threat to the safe and stable operation of surface power facilities in these $\operatorname{areas}^{[1-3]}$.

In addition to concealment and poor regularity of the general goaf, the goaf under the transmission line has the characteristics of complicated electromagnetic environment, the detection signal is easy to be affected by high-voltage electromagnetic environment and industrial stray current and so on, or be submerged in noise ${ }^{[4]}$. Traditional detection methods, such as high-density electrical method, transient electromagnetic method and so on, have single transmitted frequency, poor anti-interference ability, and rely on increasing transmitted power to improve the signal-to-noise ratio, which is easy to cause the instrument to be bulky and not conducive to field detection ${ }^{[5-7]}$.

This paper uses spread spectrum code sequence to widen the frequency of transmitted signal based on spread spectrum code technology. And a set of electromagnetic detection emission system for spread spectrum code is researched and designed to obtain better anti-interference ability by using wide spectrum and good correlation of spread spectrum code sequence, combined with spread spectrum correlation algorithm ${ }^{[8,9]}$. It provides a good reference for the development of electromagnetic detection in goaf under transmission lines.

\section{SYSTEM PRINCIPLE}

In the electromagnetic detection emission system for spread spectrum code, we assume that the transmitted signal of emission system is $x(t)$, the received signal of receiver system is $y(t)$, the response function of the measured system is $h(t)$, the response function of the transmitted signal after the measured system is $r(t)$, external noise is $n(t)$, the following relationship exists between input and output:

$$
y(t)=r(t)+n(t)=x(t) * h(t)+n(t)
$$

The two sides of the equation are correlated with the transmitted signal $x(t)$ respectively:

$$
x(t) * y(t)=x(t) * x(t) * h(t)+x(t) * n(t)
$$

There is

$$
R_{x y}(t)=R_{x x}(t) * h(t)+R_{x n}(t)
$$

Where, $R_{x y}(t)$ is the cross-correlation function of $x(t)$ and $y(t), R_{x x}(t)$ is the self-correlation function of $x(t)$, $R_{x n}(t)$ is the cross-correlation function of $x(t)$ and $n(t)$. Because spread spectrum code sequences have the characteristics similar to white noise, $x(t)$ and $n(t)$ are irrelevant, $R_{x n}(t)=0$, which can achieve the effect of noise removal and improve anti-interference ability.

\section{Design of Electromagnetic Detection Emission SYSTEM}

The electromagnetic detection emission system for spread spectrum code is mainly composed of four parts, the power supply unit, the circuit protection unit, the signal control center and the high voltage circuit. The schematic diagram of the system structure is shown in Figure I. 


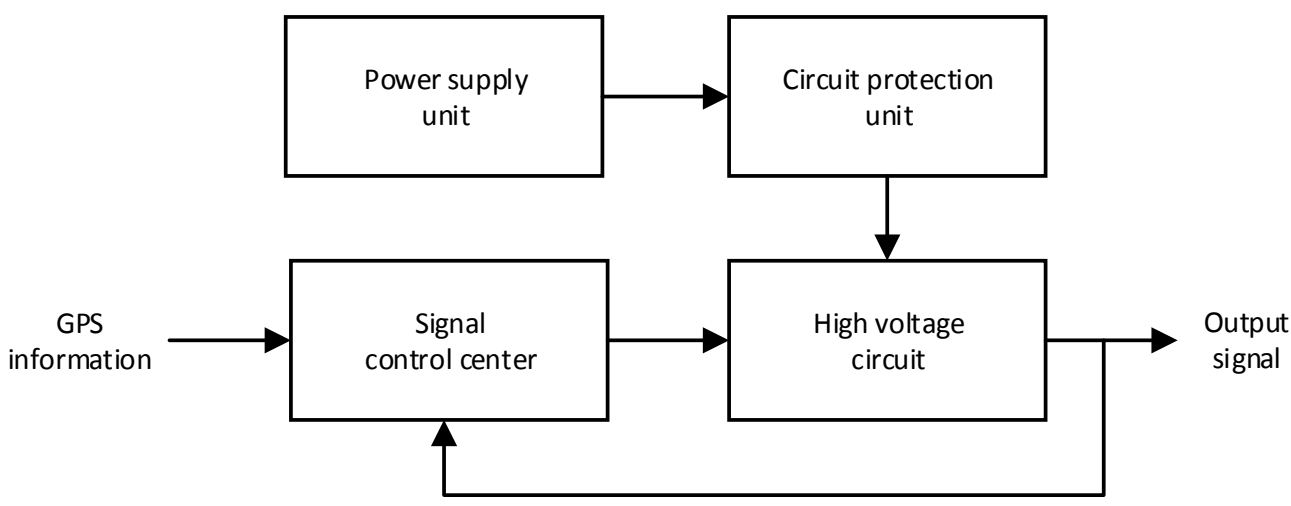

FIGURE I. SCHEMATIC DIAGRAM OF THE SYSTEM STRUCTURE

The power supply unit contains a rectifier filter circuit that converts the $\mathrm{AC}$ to $\mathrm{DC}$ and provides the power for the emission system. The circuit protection unit contains fast fuse and high voltage protection circuit to protect the emission system from over current and over voltage. The signal control center uses programmable logic device to produce spread spectrum code signal that is output to the high voltage circuit. And the GPS synchronization module is contained in the signal control center to receive the GPS information and realize the synchronous acquisition of transmitted signal and received signal, which is convenient to deal with the corresponding algorithm in the later stage. The high voltage circuit adopts optocoupler isolation circuit to realize the electrical isolation between the low voltage terminal and the high voltage terminal, which avoids the possible impact of the high voltage on the low voltage circuit and amplifies the spread spectrum code signal received from the signal control center at the same time. And the final transmitted signal is generated through the high-voltage inverter circuit. The signal control center and the high voltage circuit that are the key of the whole emission system and also the differences from other systems, will be introduced in detail below.

\section{Signal Control CENTER}

The signal control center mainly produces the spread spectrum code signal by means of Linear Feedback Shift Registers (LFSR) on the FPGA platform. In order to facilitate the relevant identification analysis in the late stage, the GPS module is used to acquire the transmitted signals and received signals at a high precision time synchronization. The schematic diagram of the signal control center structure is shown in Figure II.

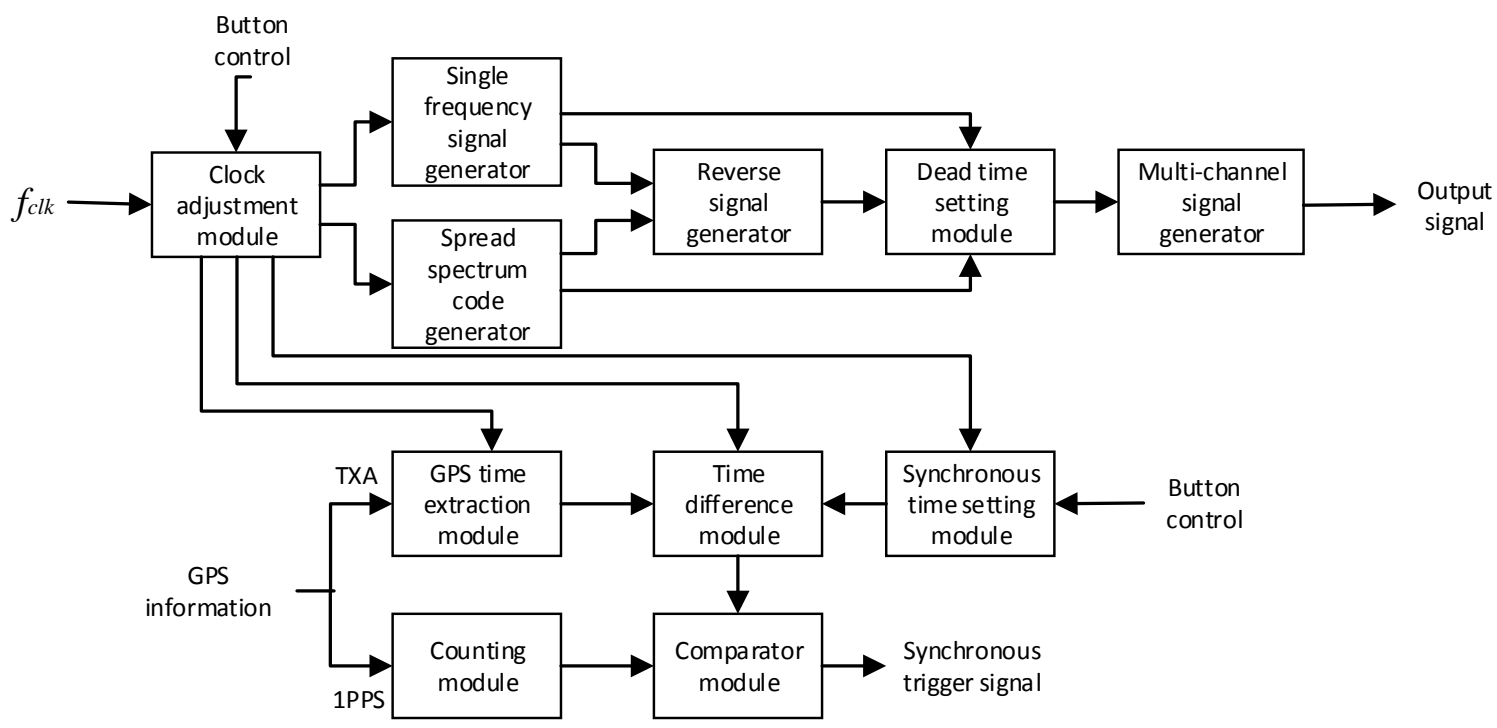

FIGURE II. SCHEMATIC DIAGRAM OF THE SIGNAL CONTROL CENTER STRUCTURE

The main frequency of the FPGA system can generate the required single frequency signal or spread spectrum signal through the clock adjustment module that can be set by users according to the requirement. The reverse signal generator can realize the reverse phase of the signal in order to drive the
IGBT module of the high voltage circuit. And the dead time setting module is added to avoid the short circuit fault when the IGBT power tubes are connected at the same time. The four signals from the multichannel signal generator can provide driving signals for the four IGBT power tubes of the 
high voltage circuit respectively. The clock adjustment module contains the GPS synchronization module that can be set the synchronization time by users and calculates the difference between the current GPS time extracted from the GPS information and the synchronization. The synchronous pulse signal of GPS is used to count and compared with the time difference, when the pulse signal and the time difference are equal, the synchronous trigger signal is output to realize the synchronous acquisition of the transmitted and received signals.

The spread spectrum code sequence is generated by LFSR, and the sequence length and the cyclic mode depend on the initial state and the feedback state of the register ${ }^{[10]}$, and the characteristic polynomial of any spread spectrum code sequence is:

$$
f(x)=c_{0}+c_{1} X+\mathrm{L}+c_{n} X^{n}=\sum_{i=0}^{n} c_{i} X^{i}
$$

Where, $n$ is the order of LFSR, the value ( 0 or 1$)$ of $C_{i}$ indicates the connection state of the feedback line, the powers of $\mathrm{x}$ is the corresponding positions of elements. LFSR can produce the corresponding spread spectrum code sequence only when $f(x)$ is the primitive polynomial. The primitive polynomial of the seven order LFSR is $f(x)=x^{7}+x^{3}+1$. The structure of seven order spread spectrum code sequence generator is shown in Figure III.

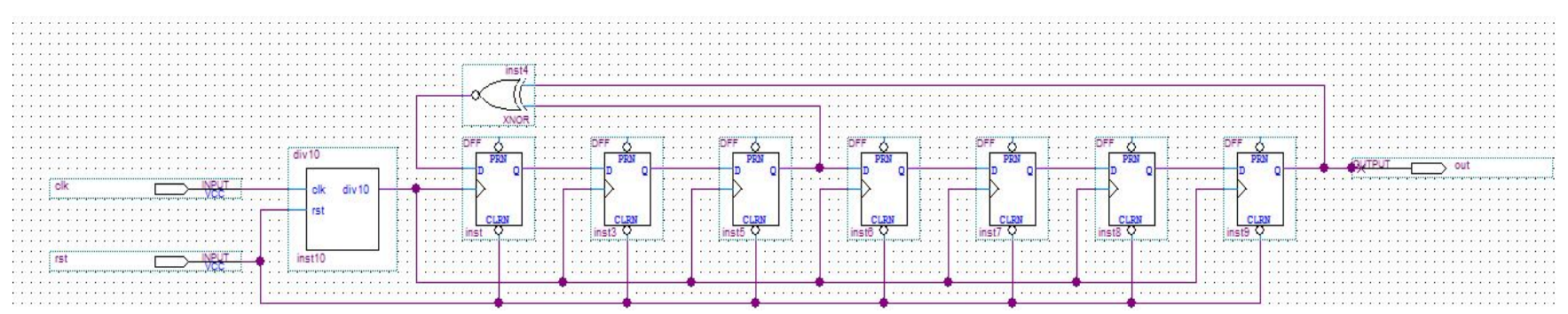

FIGURE III. THE STRUCTURE OF SEVEN ORDER SPREAD SPECTRUM CODE SEQUENCE GENERATOR

As shown in Figure III, seven D triggers that are set the same pulse signal are connected in series, the output of the third and seventh trigger is used as feedback and is connected with the input of the first flip flops, the output of the terminal trigger is the spread spectrum code sequence.

The output waveform of the seven order spread spectrum code generator is shown in Figure IV.

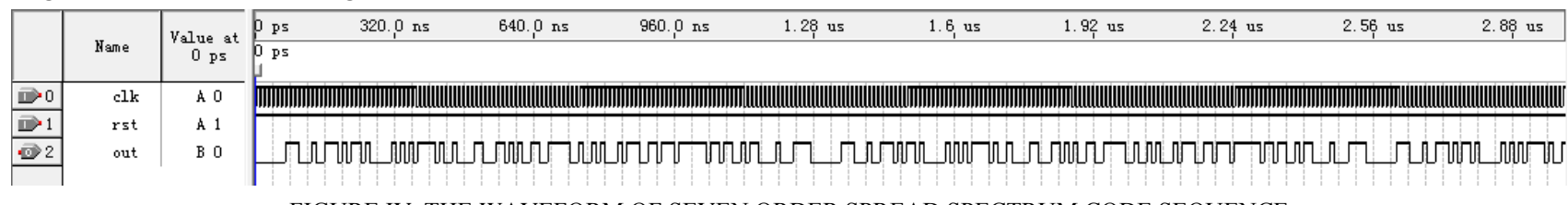

The $20 \mathrm{MHz}$ clock signal generated by the constant temperature crystal oscillator is processed by frequency division to obtain the expected frequency waveform. As shown in Figure IV, the waveform is consistent with the theoretical seven rank spread spectrum code symbol, so that the generation of spread spectrum code sequence can be realized.

FIGURE IV. THE WAVEFORM OF SEVEN ORDER SPREAD SPECTRUM CODE SEQUENCE

\section{High Voltage Circuit}

The high voltage circuit adopts the design idea of full bridge inverter circuit and uses IGBT power tube with high frequency, low loss and high voltage resistance to realize it. The schematic diagram of the high voltage circuit structure is shown in Figure V.

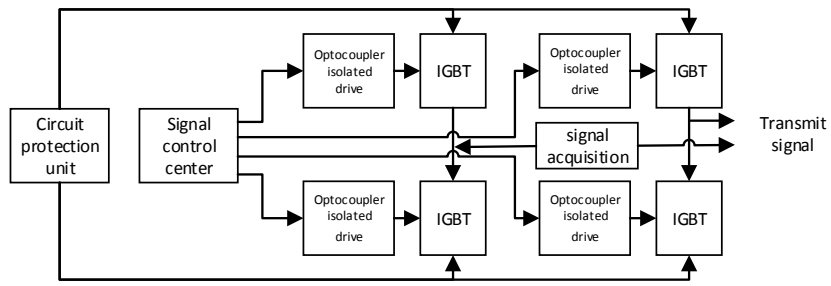

FIGURE V. SCHEMATIC DIAGRAM OF HIGH VOLTAGE CIRCUIT
The voltage and current of the circuit protection unit provide the required power for the full bridge inverter circuit. The four spread spectrum code signals generated by the signal control center drive the corresponding IGBT power tubes through the optocoupler isolation circuits, so it can produce the required transmitted signal for spread spectrum code finally.

The optocoupler isolation circuit is adopted between the signal control center and the high voltage inverter circuit to realize the signal transmission and power amplification, which avoids the lash of the high voltage of the high voltage inverter circuit to the signal control center. The schematic diagram of optocoupler isolation drive circuit and the waveforms of two optocoupler isolation drive signals are shown in Figure VI and Figure VII respectively. 


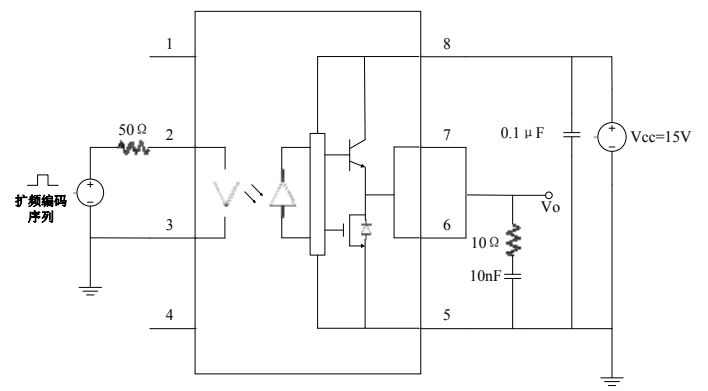

FIGURE VI. THE SCHEMATIC DIAGRAM OF OPTOCOUPLER ISOLATION DRIVE CIRCUIT

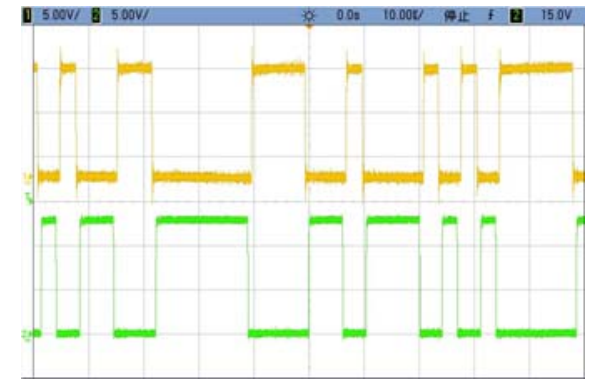

FIGURE VII. THE WAVEFORMS OF ISOLATION DRIVE SIGNALS

This emission system uses HCPLA3120 optocoupler isolation chip to design isolation drive circuit, the pin 2 is the input terminal of the spread spectrum code signal generated by the signal control center, the pin 6 and pin 7 are output terminals of the spread spectrum code signal isolated by the optocoupler, so that the IGBT power tubes connected with them can be driven without further amplifying the power.

As shown in Figure VII, the waveforms of optocoupler isolation drive signals are stable and can drive the IGBT module effectively. And there is a necessary dead time to avoid the short-circuit fault owing to the IGBT module delay caused by the reason that a bridge arm is not fully closed and another bridge arm is in the conduction state, which ensures the stable and reliable operation of the full bridge inverter circuit.

\section{ANALYSIS OF EMISSION SYSTEM OUTPUT}

The signal control center of the electromagnetic detection system for spread spectrum code is designed by programmable logic device, the high voltage circuit is designed using full bridge inverter circuit, and then the signal control center and the high voltage circuit are electrically isolated by the optocoupler isolation drive circuit that can achieve the signal transmission and amplification. So the system that is designed can produce the required signal. The output waveform of the electromagnetic detection system for spread spectrum code is shown in Figure VIII.

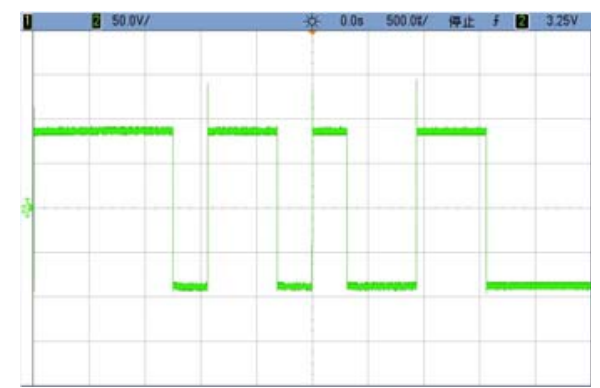

FIGURE VIII. THE OUTPUT WAVEFORM OF THE ELECTROMAGNETIC DETECTION SYSTEM

As shown in Figure VIII, the waveform is stable and has the required pseudo random property. It can avoid the interference from electromagnetic environment very well combined with the correlation identification method. At the same time, the wide frequency range can obtain more abundant detection data and information in limited detection time, so as to improve detection efficiency and accuracy.

\section{CONCLUSION}

In view of the complicated electromagnetic environment in the goaf under transmission lines and the traditional detection methods have the disadvantages such as single transmission frequency, low detection efficiency, increasing transmitted power to improve the signal to noise ratio and equipment bulky, etc. In this paper, a set of electromagnetic detection emission system for spread spectrum code has been researched and designed referring to the spread spectrum coding technology in communication field. It can effectively improve detection efficiency and accuracy combined with spread spectrum code correlation identification method, provide reference for power grid planning, erection and have important practical significance for ensuring the safe and stable operation of power grid.

\section{ACKNOWLEDGMENT}

Tanks NSFC(51677181), and National Power Grid Corp science and technology project(52053016000X).

\section{REFERENCES}

[1] Zhang J Q, Yang K, Wang Y D, et al. Research on Foundation Treatment of High Voltage Transmission Towers Erected above Goaf of Coal Mine[J]. Power System Technology, 2006, 30(2):30-34.

[2] Zhimei Chen, Guanglin Yuan, Kang Song. Safety Evaluation of High Voltage Transmission Lines in Coal Mining Areas, China Electric Power[J] , 2015, 48(10):101-106.

[3] Yang F L, Yang J B, Han J K, et al. Bearing Capacity Computation of UHV Transmission Tower With Foundation Deformation Above Coaf of Goal Mine[J]. Proceedings of the Csee, 2009, 29(1):100-106.

[4] Zhao L, Lu J, Wu G. Measurement and Analysis on Electromagnetic Environment of $1000 \mathrm{kV}$ UHV AC Transmission Line[C]// Asia-Pacific Power and Energy Engineering Conference. IEEE, 2012:1-4.

[5] Huang Q. The application of High Density Resitivity Method and Transient Electromagnetic Method to the detection of coal mine goaf[J]. Geophysical \& Geochemical Exploration, 2012.

[6] Guoqiang Xue, Shu Yan, Qingyun Di. Technical Analysis of Multitransient Electromagnetic Method [C], Institute of Geology and Geophysics, Chinese Academy of Sciences, 2015, 2016.

[7] Qiao Y, Zhang Z. The application of Transient Electromagnetic Method in detection of coal mine goaf[C]// IEEE International Conference on 
Spatial Data Mining and Geographical Knowledge Services. IEEE, 2011:574-576.

[8] Wu Huang. Study on the frequency interference spectrum of electromagnetic instrument[J]. Engineering Technology: 2015(13):00241-00242.

[9] Shiqiang Li, Guoqiang Liu, Hui Xia, Yanhong Li, Research on the Waveform Synthesis of Electromagnetic Detection Inverter based on FPGA, Advanced Materials Research, 2012, 433-440: 5628-5635.

[10] Walczak J, Stępień R. The method of improving pseudo random signal generating rate of the LSFR generators[J]. Istet International Symposiumon Theoretical Electrical Eng ineering, 2013. 